\title{
Maxillary Anterior Segmental Distraction Osteogenesis (MASDO) for Cleft Patients
}

\author{
Mikihiko KOGO, DDS, $\mathrm{PhD}^{* 1)}$, Tomonao AIKAWA, DDS, $\mathrm{PhD}^{1)}$, Takeshi HARADA, DDS, $\mathrm{PhD}^{1)}$, \\ Kohji ISHIHAMA, DDS, $\mathrm{PhD}^{1)}$, Masataka HIGUCHI, DDS, $\mathrm{PhD}^{1)}$, Ayako USHIMURA, DDS, $\mathrm{PhD}^{1)}$, \\ Masaya OKURA, DDS, $\mathrm{PhD}^{1)}$ and Seiji IIDA, DDS, $\mathrm{PhD}^{2)}$
}

\begin{abstract}
Maxillary anterior segmental distraction osteogenesis (MASDO) is an effective surgical orthodontic treatment for reduced maxilla in cleft lip and palate because of its benefit of conserving velopharyngeal function. In this review article, we summarize the procedure and present two cases of MASDO treatment using a bone-borne distractor and a hybrid-type distractor.
\end{abstract}

\section{Introduction}

The aims of treatment for patients with cleft lip and palate are to obtain competent velopharyngeal function, good occlusion, and good aesthetic appearance. Thus, all treatments for cleft lip and palate should be considered and an adequate balance achieved among them. Of these, velopharyngeal function is very important for speech, communication, and social life. Velopharyngeal function is assessed by findings of naso-pharyngeal fiberscope, soft palate position on lateral radiograms, and nasality, and then diagnosed as velopharyngeal competence (VPC), velopharyngeal incompetence (VPI), or borderline. These examinations and speech treatments should be continued until adulthood to obtain normal speech function.

In general, palatoplasty operations to close the palatal cleft and to construct velopharyngeal muscle are performed at around 1 to 1.5 years of age in order to obtain sufficient speech function. This palatoplasty operation to the developing maxilla, however, inhibits postoperative maxillary growth to varying degrees ${ }^{1)}$. Thus, the management of occlusion and jaw growth of cleft patients sometimes require orthognathic surgical intervention.

The introduction of distraction osteogenesis (DO) has increased the therapeutic variation of orthognathic surgery for cleft patients, especially for those cases with severe maxillary retrusion and morphological problems of maxillary bone ${ }^{2)}$. Among DO operations, maxillary advancement combined with Le Fort I osteotomy (LF1 DO) has become one of the standard treatments for severe maxillary retrusion of cleft lip and palate patients, because LF1 DO has the advantage of less damage to velopharyngeal function and allows a larger amount of maxillary advancement, compared with LF1 osteotomy ${ }^{2}$. However, even in patients who have undergone the LF1 DO advancement procedure, surgical intervention may worsen the velopharyngeal function of patients who are on the borderline between VPC and VPI. Furthermore, LF1 DO with an internal distractor suffers limitations of movement vector ${ }^{3)}$ such as rotational movement. Moreover, LF1 DO using an external distractor, which allows complicated 3dimensional movement, causes mental stress for patients because they must wear a large head-mounted external distractor for long periods ${ }^{1)}$.

Maxillary anterior segmental distraction osteogenesis (MASDO) is a new procedure of orthognathic surgery to produce an ideal maxillary dental arch form in such patients. The features of MASDO are as follows: 1) a distraction segment is made at the anterior part of

1) Professor and Chair*, First Department of Oral and Maxillofacial Surgery, Osaka University Graduate School of Dentistry, 1-8, Yamadaoka, Suita, Osaka, 565-0871, Japan

2) Professor, Department of Oral and Maxillofacial Reconstructive Surgery, Okayama University Graduate School of Medicine, Dentistry, and Pharmaceutical Science, 2-5-1, Shikata-cho, Kita-ku, Okayama, 700-8525, Japan 
the maxilla, 2) an anterior segment is advanced anteriorly using an intraoral device, 3) the midline of the maxillary incisor can be adjusted to the facial midline by straight or rotational movement of the segment, 4) the posterior part of the maxilla is preserved and velopharyngeal function is not affected by this procedure $^{4,5}$. In our department, MASDO has been performed for severely retruded maxillary dental arches using a single palatal distractor, and successful results were obtained in both aesthetic and functional aspects. In this original procedure, the segment was anteriorly advanced in a straight line, in which the vector was determined by the position of the palatal distractor ${ }^{4)}$. Then, we started to use two distractors, placed at the buccal side adjacent to the alveolar osteotomy line. In this procedure, the anterior segment was advanced with rotational movement, by changing the amount of deviceactivation between the two distractors ${ }^{5)}$.

In this review article, we explain the treatment procedures and management of MASDO for maxillary retrusion patients with cleft lip and palate, and present two cases: one is straight movement of the segment, and the other is rotational movement.

\section{Outline of MASDO}

\section{Indications}

Patients with complete cleft palate who have a severely retruded malpositioned anterior maxillary bone would be indicated for MASDO by our procedure. Secondary alveolar bone grafting should be performed in advance. From April 2003 to August 2011, we have performed MASDO operations for patients with complete cleft lip and palate at the First Department of Oral and Maxillofacial Surgery, Osaka University Dental Hospital. Age at operation ranged from 14 to 36 years old, with a mean age of 19.3 years old. The cleft type of 31 patients was unilateral cleft lip and palate $(\mathrm{n}=23)$ and bilateral cleft lip and palate $(\mathrm{n}=8)$.

\section{Surgical procedure}

For planning the MASDO treatment, model surgery is necessary, and the design of osteotomy and amount of segment movement should be discussed with orthodontists (Fig. 1A, B). MASDO surgery is performed under nasoendotracheal anesthesia. An incision is made based on the principle of maxillary osteotomy procedure for cleft patients so that blood supply to maxillary bone from the surrounding membrane can be maintained. For MASDO using a palatal distractor, a vestibular vertical incision is made to expose the buccal osteotomy line, and for MASDO using two buccal distractors, a horizontal vestibular incision is made. Then, minimum incisions are made on the palatal mucosa along the gingival sulcus, and a palatal mucoperiosteal flap tunnel is made to expose the palatal osteotomy line (Fig. 1C). Before osteotomy, the distractor is adjusted and positioned, and then drill holes are made. At this point, bone screws should not be inserted into the bones, to avoid repeated insertion of screws. Then osteotomy is conducted in accordance with the planned lines using a small surgical reciprocating saw and a thin chisel (Fig. 1D). Then, separation of the nasal septum from the nasal base is conducted, followed by down fracture of the anterior segment. During these procedures, care must be taken to prevent damage to the palatal mucosa, palatal artery, and nasal mucosa.

The DynaForm System (Stryker Osteosynthesis, Leibinger Micro Implants, Kalamazoo, MI) is used as the distractor. With the anterior segment being mobilized, the distractor is re-positioned on the drilled holes. In MASDO using a single distractor on the palate, bone screws are inserted transmucosally with an angled driver (Fig. 1E and 2A).

On the other hand, in MASDO using two distractors on the buccal side (Fig. 2), the two arms appearing in the oral cavity are adjusted to set along the teeth on each segment; one is on the distraction segment and the other is on the posterior segment. Then, the two arms located on the bone surface are adjusted to set on each segment. Screw rings are set at the tips of all arms. The devices are secured by two bone-screws on the maxillary bone segment, and are wire-ligated with two teeth on each segment. This wire-ligation is further fixed with quick-cure resin (Fig. 2B).

\section{Activation of devices and postoperative manage- ment}

After a 7-day latency period, the distractors are activated $0.5 \mathrm{~mm}$ twice a day $(1.0 \mathrm{~mm}$ per day) (Fig. $1 \mathrm{~F})$. In MASDO using a palatal distractor, orthodontic elastic protraction with a face mask is simultaneously supplemented to support anterior traction force and to prevent upward movement due to soft tissue tension. If necessary, maxillo-mandibular elastic tractions are 

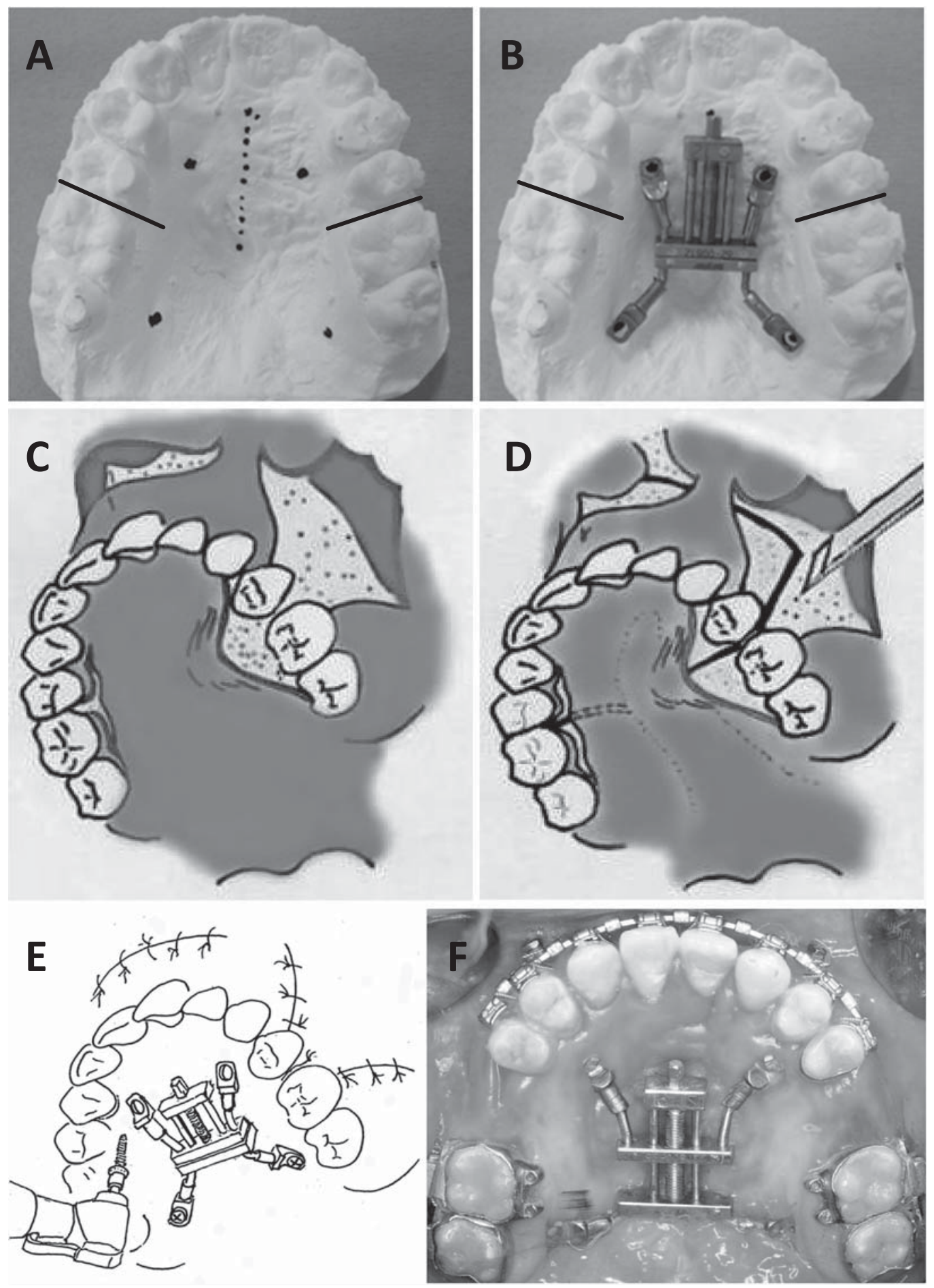

Fig. 1 Schematic diagram of preparation and operation procedure.

A : Determination of the direction for advancement (dotted line), positions of fixing screws (dot) and alveolar osteotomy line (solid line) on dental cast model.

B : Adjustment of the distractor on the dental cast model.

C : An incision line and exfoliated area should be made to supply blood to the maxillary bone segment from the surrounding membrane.

D : Osteotomy is performed by using a surgical saw and chisel. The alveolar osteotomy line is located in the space between the premolar and molar.

$\mathrm{E}$ : The distractor is fixed by bone screws, longer than $10 \mathrm{~mm}$, which are transmucosally inserted using an angled driver.

$\mathrm{F}$ : Intraoral findings during advancement. After a 7-day latency period, the distractor is activated by $1.0 \mathrm{~mm} / \mathrm{day}$. 

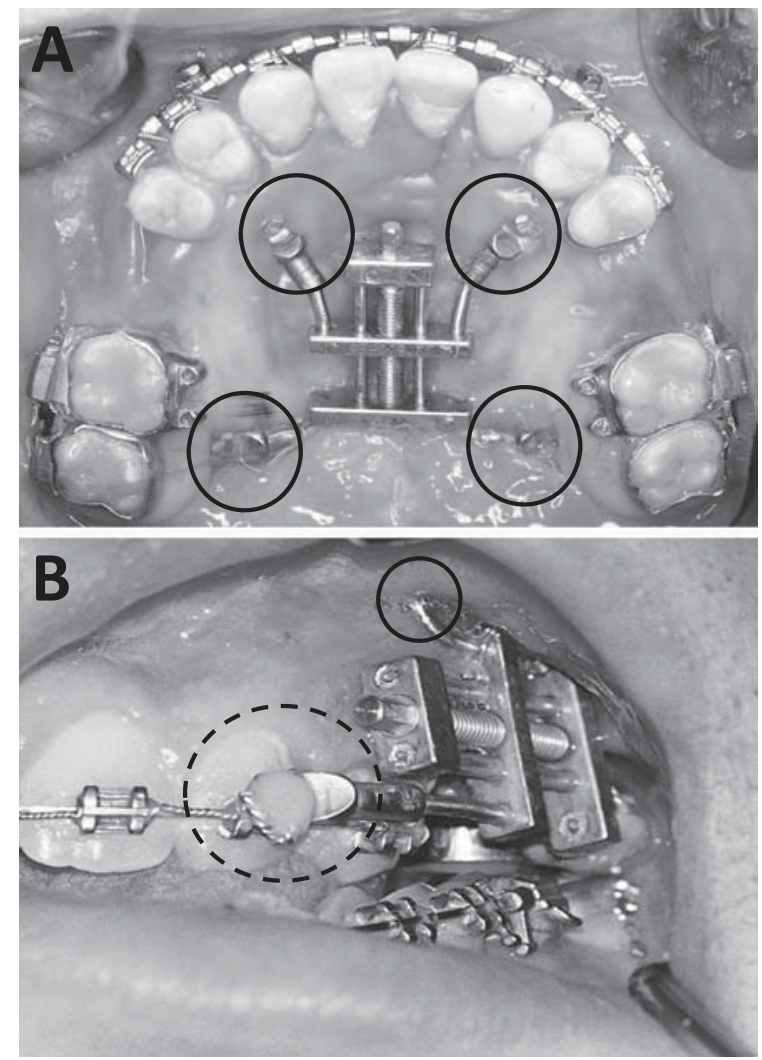

Fig. 2 Two types of applying the distractor (The DynaForm System $\left.{ }^{\circledR}\right)$.

A : Bone-borne appliancec the distractor is fixed by bonescrews on each segment (solid circle).

B : Hybrid type appliance; the distractor is fixed by bonescrews and wire-ligation with teeth; this is a hybrid of the bone-borne appliance (solid circle) and tooth-borne appliance (dotted circle).

used for minor correction of the position of the segment. After completion of distraction and correction of the segment, anterior segment teeth are connected to posterior segment molar teeth with orthodontic wire to fix the position. After a retention period of 2 to 3 months, radiographic examination should be performed to ensure de-novo bone formation at the distraction space, and then the distractors can be removed under local anesthesia. Patients should be instructed to keep wearing the face mask at home and at night for at least 3 months after distraction has been completed. In MASDO using buccal distractors, the segment can be rotated by using different activating amounts in the two distractors to adjust the maxillary midline to the facial midline and to correct the dental arch form. After completion of the planned advancement on each side, surgeons and orthodontists should estimate the result of advancement and assess the orthodontic elastic necessity of additional protraction or guidance by maxilla-mandibular elastic traction for further correction. After a retention period of 2 to 3 months, the distractors can be removed under local anesthesia, and then the anterior segment teeth can be connected to the posterior segment molar teeth with orthodontic wire to fix the position.

\section{Case reports}

\section{Case 1}

An 18-year old Japanese male with complete bilateral cleft lip and palate. His surgical treatment histories were as follows: primary cheiloplasty with twostage procedure at the age of 4 months and 7 months, palatoplasty with push-back procedure at 1.5 years old, osteotomy and repositioning of premaxillary bone with secondary bone grafting of the alveolar cleft at 13 years old, and pharyngeal flap operation at 16 years old. All surgeries were performed at our department. The diagnosis was Class 3 malocclusion with overjet of $-6.0 \mathrm{~mm}$ due to maxillary hypoplasia. Examination of velopharyngeal function revealed the risk of speech deterioration due to the surgical intervention of LF1 maxillary advancement. Thus, MASDO, with a bone-borne distractor positioned on the palate, was performed for surgical correction of the deformed small maxilla. After a 7-day latency period postoperatively, the distractor was activated by $1.0 \mathrm{~mm} /$ day. Maxillary correction was achieved by linear advancement movement of the anterior maxillary segment of $10.0 \mathrm{~mm}$. Velopharyngeal function remained the same as the pre-operative status, and after a retention period of 2 months, the distractor device was removed. Two years after MASDO, a dental implant was inserted in each distraction gap, and an ideal maxillary dental arch form and occlusion relationship were achieved (Fig. 3).

\section{Case 2}

A 22-year-old Japanese male with left-side unilateral left cleft lip and palate. His surgical treatment histories were as follows: primary cheiloplasty at the age of 3 months, palatoplasty with push-back procedure at 13 months, and secondary bone grafting of the alveolar cleft at 9 years old. These operations were performed at another institution. The diagnosis was Class 3 malocclusion with overjet of $-18.0 \mathrm{~mm}$ due to maxillary 

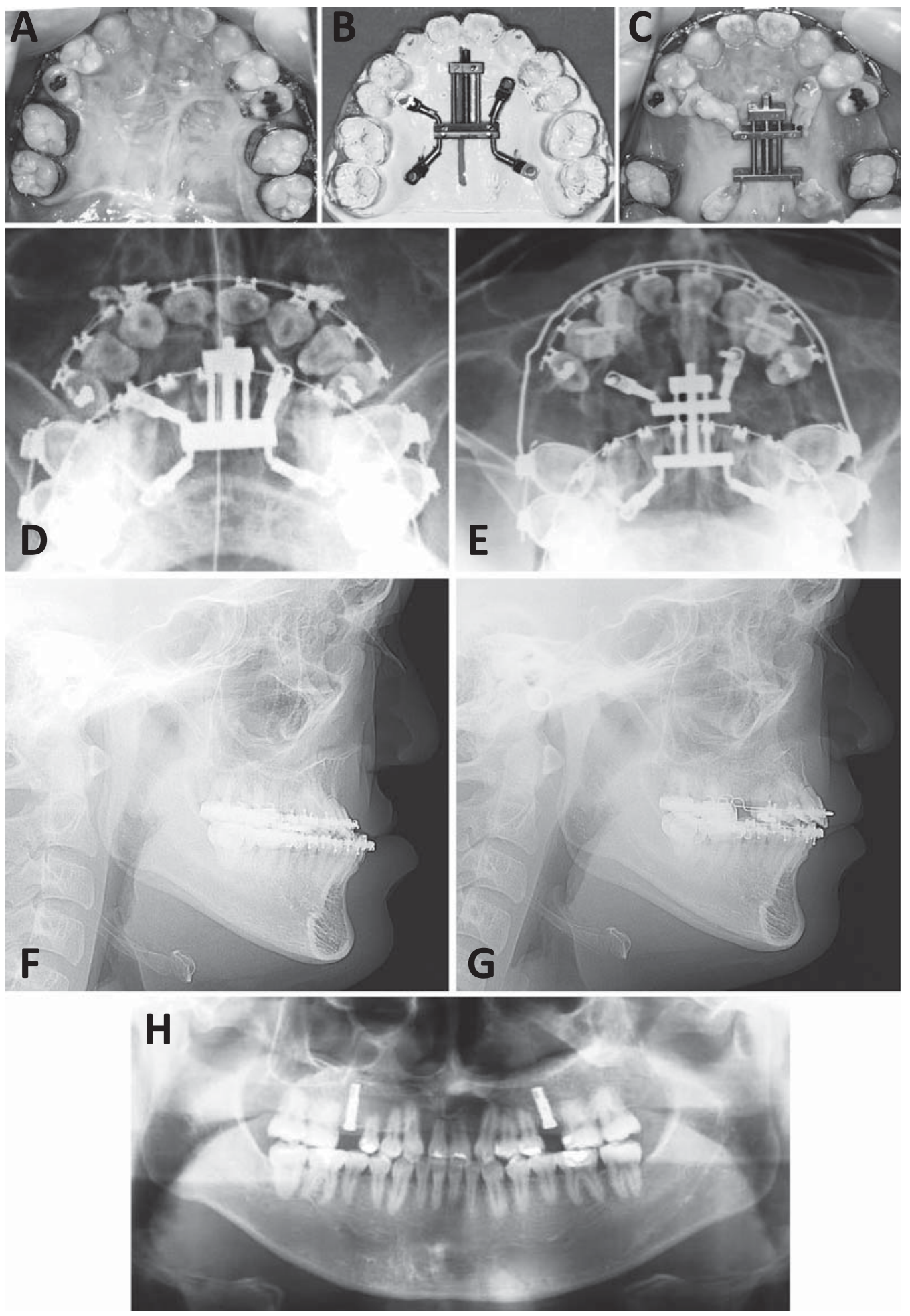

Fig. 3 Case 1

A : Intraoral finding before operation.

B : Adjustment of the distractor on dental cast model.

C : Intraoral finding after the completion of advancement.

D : Axial radiograph image immediately after operation.

$\mathrm{E}$ : Axial radiograph image after the completion of advancement.

$\mathrm{F}$ : Lateral cephalometric radiograph before operation.

G: Lateral cephalometric radiograph one year after advancement.

$\mathrm{H}$ : Panoramic radiograph after implant surgery. 
retrusion and mandibular protrusion. Examination of nasopharyngeal function revealed the risk of speech deterioration due to LF1 maxillary advancement of more than $5 \mathrm{~mm}$. His maxillary dental arch was rotated to the right, and the U1-midline was deviated to the right. Thus, MASDO, with two hybrid-type distractors positioned on the bilateral alveolar, was performed for surgical correction of the deformed small maxilla and to adjust the maxillary midline to the facial midline as much as possible. After a 7-day latency period postoperatively, the distractor was activated by $1.0 \mathrm{~mm} /$ day. Maxillary correction was achieved by rotational advancement movement of the anterior maxillary segment, with $12.0 \mathrm{~mm}$ on the right side and $3.9 \mathrm{~mm}$ on the left side. Because his orthodontist planned a two-stage surgical treatment for correction of the maxillo-mandibular deformity, presurgical orthodontic treatment for mandibular osteotomy has been continuing (Fig. 4).

\section{Discussion}

Patients with cleft lip and palate always have some disadvantages of maxillary growth and deformity of the maxillary dental arch to varying degrees because of post-operative surgical scar contraction. Such patients also have the disadvantage of velopharyngeal dysfunction. To improve the aesthetic profile and functional occlusal relationship, maxillary advancement is often considered. After a suitable palatoplasty operation, the levator veli palatini muscle stays off the posterior border of the maxilla and palatine bone, and post-operative scar tissue exists between the muscle and posterior border of the maxilla. Thus, maxillary advancement always affects the position of the levator veli palatini muscle and velopharyngeal function. This disadvantage should be considered when planning the treatment.

In our hospital, velopharyngeal function is routinely assessed by speech examination, nasality, cephalometric radiograms, and nasoendoscopy examination for patients who may undergo maxillary advancement. Nasality is assessed by speech and a nasometer. Cephalometric radiograms with resting status, /a/ phonation, and forced blowing are used for evaluating soft palate movement and the distance from the posterior wall of the pharynx to the soft palate. Nasoendoscopy examination determines the velopharyngeal status and reveals the most reliable status of velopharyngeal function because not only soft palate motion but also pharyngeal wall motion to close the velopharyngeal orifice can be visualized. Furthermore, air-bubble formation during the /p/phonation, which is easily assessed as the borderline of VPC and VPI, can be visualized, too ${ }^{6)}$.

We developed the MASDO procedure for those cleft patients on the borderline or with VPI, to advance the maxilla without worsening the velopharyngeal function $^{4,5)}$. The distraction segment of MASDO is made in a position anterior to the first molar teeth. Thus, the posterior border of the maxilla, levator veli palatine muscle, and scar tissue between them are intact after the osteotomy and distraction procedure. We have performed MASDO in 31 cases to advance retruded maxilla for patients with cleft lip and palate, and the velopharyngeal function of all the patients examined was no worse after the MASDO treatment.

We have used two methods to fix the distractor for MASDO procedures. One is to fix a device with four bone-screws on the palate, the so-called bone-borne appliance (Fig. 2A). The other is to fix distractor (s) with two bone-screws and two wire-ligations to teeth, and is a combination of bone-borne appliance and toothborne appliance, the so-called hybrid-type distractor (Fig. 2B). Linear advancement was achieved by simply activating the palatal distractor (Fig. 2A and Fig. $3)$. The rotational movement of the segment can be achieved by different amounts of advancement at the osteotomized area on each side (Fig. 2A and Fig. 4). We performed MASDO in 31 cases from April 2003 to August 2011. Of these, the bone-borne type of palatal single distractor was used for 18 cases, and the hybrid type of buccal distractor was used for 13 cases. The average amount of linear advancement using palatal distractors was $10.4 \mathrm{~mm}$. On the other hand, the amounts of advancement differed for each buccal hybrid type of distractor: the average of the smaller side was $4.7 \mathrm{~mm}$, ranging from 0 to $6 \mathrm{~mm}$, and that of the larger side was $8.9 \mathrm{~mm}$, ranging from 6 to $12 \mathrm{~mm}$. The planned maxillary advancement was achieved in all the cases.

The MASDO procedure produced varying amounts of distraction space. These spaces were used (will be used) as follows: 1) orthodontic alignment of crowded teeth, 2) orthodontic anterior movement of posterior molar teeth, and 3) prosthodontic treatment including 

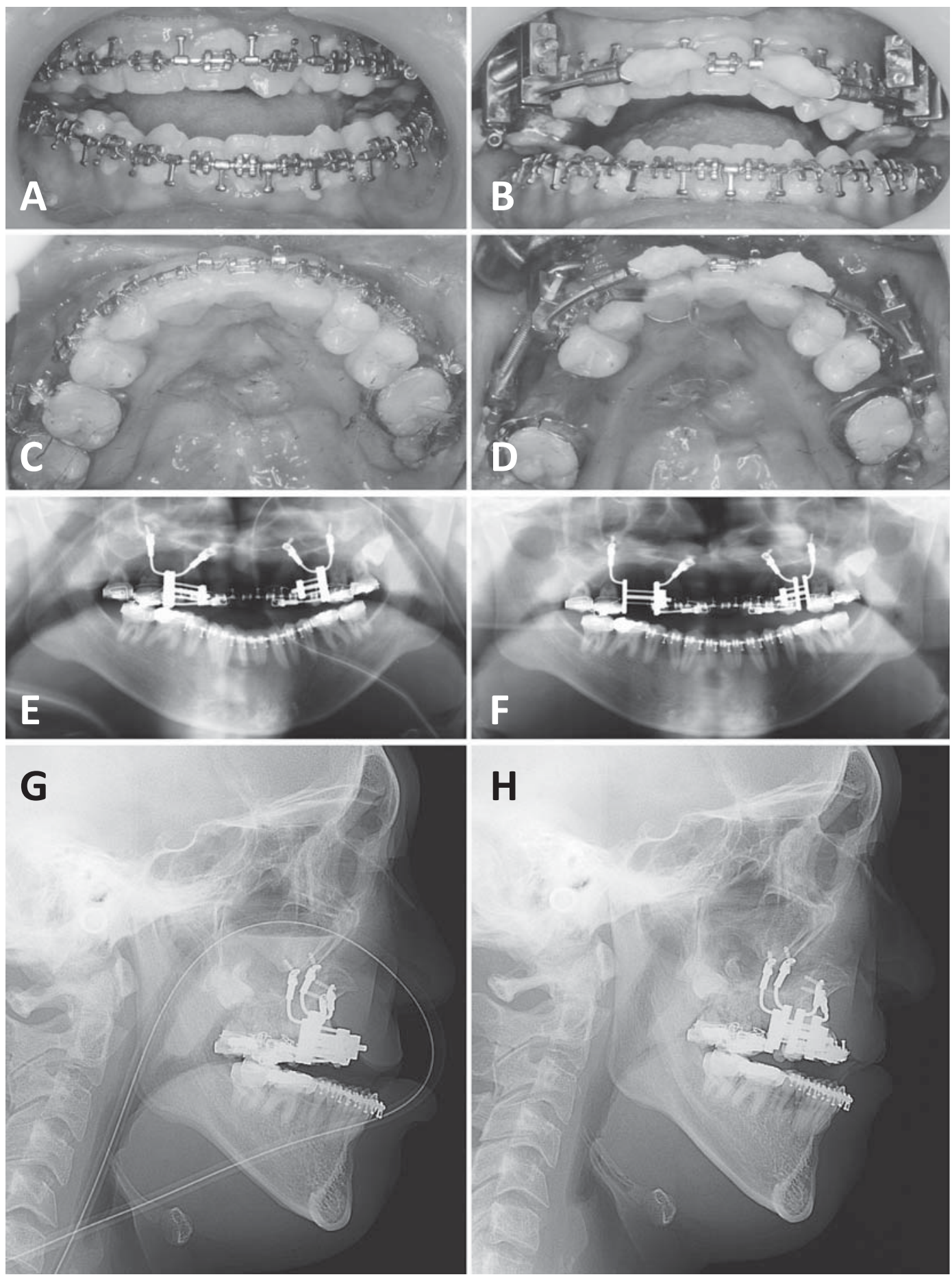

Fig. 4 Case 2

A and $\mathrm{C}$ : before operation, $\mathrm{E}$ and $\mathrm{G}$ : immediately after operation, B, D, F and H: after the completion of advancement.

A and B: occlusion, $\mathrm{C}$ and D: maxillary dental arch, E and F: panoramic radiograph, $\mathrm{G}$ and $\mathrm{H}$ : lateral cephalometric radiogram.

dental implants. By MASDO surgery and postoperative treatment of the distraction space, occlusion function and aesthetic problems were improved in 12 of the 31 cases. In the remaining 19 cases, further orthognathic surgeries were (will be) required. Of these, sagittal splitting ramus osteotomy (SSRO) was (will be) required 
for 11 cases, two jaw surgeries (Le Fort I osteotomy and SSRO) were (will be) required for 7 cases, and maxillary corticotomy was required for one case. Although these features are one of the demerits of MASDO, MASDO may be a good therapeutic option to obtain aesthetic improvement and a good occlusal relationship with no risk of worsening the speech function. The merits and demerits of MASDO treatment are as follows:

Advantages :

- Advance maxilla without affecting speech

- Enlarge maxillary length

- Make space to align dislocated teeth

- Possible to align molar teeth anterior

- Enable anterior segment rotation

Disadvantages and limitations

- Difficulty of vertical control of molar teeth

- Difficulty of cant control

- Risk of root injury (none/31 cases)

- Additional surgery (19/31 cases)

\section{Reference}

1) Kitagawa, T., et al.: Dentoalveolar growth of patients with complete unilateral cleft lip and palate by early two-stage furlow and push-back method: preliminary results, Cleft Palate Craniofac J, 41 : 519-525, 2004.

2) Scolozzi, P., et al.: Distraction osteogenesis in the management of severe maxillary hypoplasia in cleft lip and palate patients, J Craniofac Surg, 19 : 1199-1214, 2008.

3) Aikawa, T., et al.: Breakage of internal maxillary distractor: considerable complication of maxillary distraction osteogenesis, Oral Surg Oral Med Oral Pathol Oral Radiol Endod, 106 : e1-7, 2008.

4) Iida, S., et al.: Maxillary anterior segmental distraction osteogenesis with the dynaform system for severe maxillary retrusion in cleft lip and palate, Plast Reconstr Surg, 120 : 508-516, 2007.

5) Aikawa, T., et al.: Rotation movement of the anterior maxillary segment by hybrid distractor in patients with cleft lip and palate, Oral Surg Oral Med Oral Pathol Oral Radiol Endod, 110 : 292-300, 2010.

6) Matsuya, T., et al.: A fiberscopic study of velopharyngeal closure in patients with operated cleft palates, Plast Reconstr Surg, 63 : 497-500, 1979. 